\title{
AvanCES EN PETROgRAFÍA AUTOMATIZAdA: CERÁMICAS TEMPRANAS DE Guatacondo, norte de Chile (900 A.C-200 D.C.)
}

\author{
Camila Riera-Soto ${ }^{2}$ \\ Mauricio Uribe Rodriguez ${ }^{\mathrm{b}}$ \\ Andrew Menzies ${ }^{\mathrm{c}}$ \\ Monserrat Barraza Bustos ${ }^{\mathrm{d}}$
}

\begin{abstract}
Resumen
El estudio petrográfico mediante QEMSCAN de fragmentos cerámicos de la Región de Tarapacá, pertenecientes al Periodo Formativo Temprano (900 a.C.-200 d.C.), evidencia distintos orígenes mineralógicos del material cerámico, ya sea por producción local, circulación o intercambio de alfarería en el norte de Chile. En Guatacondo, la morfología, características de superficie y el análisis macroscópico de pastas permitieron avanzar en el conocimiento acerca de los tipos cerámicos tempranos LCA y LMS del norte chileno. En esta oportunidad, los resultados de QEMSCAN confirman la existencia de grupos petrográficos concordantes con ambos tipos estilisticos y los estudios descriptivos mencionados. A partir de las muestras de Guatacondo se puede reconocer la convergencia de tradiciones diferentes en la selección de materias primas y producción de las cerámicas iniciales para esta región. La evidencia petrográfica es indicativa de la producción, circulación, intercambio de vasijas y/o conocimientos a escala local o larga distancia. Por lo tanto, el conocimiento detallado que ofrece esta técnica junto con los análisis tipológicos previos, permite precisar la variabilidad de estas tecnologias cerámicas, así como visualizar las posibles áreas de aprovisionamiento de materias primas en una época inicial del desarrollo alfarero en los Andes Centro Sur.
\end{abstract}

Palabras clave: cerámica, petrografia automatizada, QEMSCAN, Periodo Formativo Temprano, Tarapacá

Abstract

\section{ADVANCES IN AUTOMATED PETROGRAPHY: EARLY CERAMICS OF GUATACONDO, NORTH OF CHILE (900 BC-200 AD)}

The petrographic study by QEMSCAN of ceramic fragments of the Tarapaca Region, belonging to the Early Formative period (900 BC-200 AD), evidence different mineralogical origin of the ceramic material, either by local production, circulation or pottery exchange in the north of Chile. In Guatacondo, the morphology, surface characteristics and the macroscopic analysis of ceramics pastes allowed to advance in the knowledge about the early ceramic types LCA and LMS

a Programa de Postgrado en Antropología, mención Arqueología, Universidad Católica del Norte, San Pedro de Atacama, Chile

Correo electrónico: cami.riera.soto@gmail.com

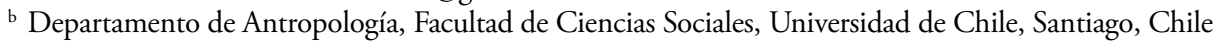
Correo electrónico: mur@uchile.cl

c Departamento de Ciencias Geológicas, Universidad Católica del Norte, Antofagasta, Chile

Correo electrónico: amenzies@ucn.cl

d Maini, Unidad de Equipamiento Científico, Universidad Católica de Norte, Antofagasta, Chile Correo electrónico: mbarraza@ucn.cl 
of the Chilean north. In this opportunity, the results of QEMSCAN confirm the existence of petrographic groups concordant with both stylistic types and the descriptive studies mentioned. From the Guatacondo samples, we can recognize the convergence of different traditions in the selection of raw materials and production of the initial ceramics for this region. The petrographic evidence is indicative of the production, circulation, exchange of vessels andlor knowledge at local or long-distance scales. Therefore, the detailed knowledge offered by this technique, together with the previous typological analyzes, allow us to specify the variability of these ceramic technologies, as well as to visualize the possible areas of supply of raw materials in an initial period of the pottery development in the South-Central Andes.

Keywords: Ceramic, automated petrography, QEMSCAN, Early Formative Period, Tarapacá

\section{Introducción}

El estudio petrográfico de pastas cerámicas es uno de los mejores caminos para conocer las fuentes de materias primas con que las culturas del pasado se aprovisionaban. Las inclusiones no plásticas y la matriz arcillosa nos acercan al contexto geológico en el que se encuentran las fuentes probables. Por lo tanto, la descripción de mineralogía y morfología de las inclusiones no plásticas, proporcionan información sobre las formaciones geológicas que les dieron origen, así como los procesos naturales y culturales que llevaron a estas inclusiones y la matriz, a ser partes de una vasija. En este artículo se presentan los resultados del análisis petrográfico realizado a fragmentos cerámicos correspondientes al Período Formativo Temprano, recolectados en la localidad de Guatacondo en la región de Tarapacá, norte de Chile, en la subárea de Valles Occidentales, Andes Centro Sur (Fig. 1). El análisis petrográfico se basó en el análisis mineralógico automatizado mediante QEMSCAN, correspondiente a una innovación tecnológica para el estudio de pastas de cerámicas arqueológicas (Menzies et al. 2015).

La región de Tarapacá se ubica entre la quebrada de Camarones, al Norte, y la cuenca del río Loa, al Sur. La localidad de Guatacondo (Fig. 1) es parte de la quebrada del mismo nombre y corta porciones de las secuencias sedimentarias del Neógeno y Cuaternario que constituyen la Pampa del Tamarugal, un rasgo geológico característico del sector norte del Desierto de Atacama en Chile. En su mayoría, las secuencias sedimentarias corresponden a llanuras aluviales que tienen su punto de origen en la cadena montańosa Sierra de Moreno, compuesta por una superposición de rocas metamórficas del Proterozoico, secuencias sedimentarias del Jurásico hasta el Cuaternario, secuencias volcánicas del Mioceno y cuerpos intrusivos del Cretácico al Paleoceno (Blanco y Tomlinson 2013). Por lo tanto, se trata de una zona con una gran variedad de fuentes de materias primas, las que pueden variar en distancias cortas.

Hasta hace poco, las investigaciones cerámicas en Tarapacá habían sido pocas y de modo intermitente (entre otros, Núñez y Moragas 1983; Mavrakis 1985; Rivera 1988-89; Núñez y Santoro 2011), es por ello que la reconstrucción de la prehistoria regional es una tarea aún pendiente. En lo que respecta a los estudios de cerámicas arqueológicas formativas, los análisis más actuales en las regiones de Tarapacá y Atacama, se incluyen los avances de Sinclaire y colaboradores (1998), Uribe y Ayala (2000), Uribe (2004, 2006, 2009), Uribe y Vidal (2012, 2015), Uribe y colaboradores (2019), entre otros. Estos trabajos detallados dejan atrás el criterio tipológico y el material funerario anteriores, dirigidos principalmente a establecer secuencias histórico-culturales. Lo anterior, gracias a que la región de Tarapacá presenta un desarrollo temprano y continuo de sistemas de asentamiento complejos, junto con evidencias de innovaciones tecnológicas que apuntan a cambios sociales notorios en el registro arqueológico en torno al primer milenio antes de esta Era.

Justamente, Uribe y Vidal $(2012,2015)$ y Uribe y colaboradores (2019) han proporcionado estudios sistemáticos y actualizados del material cerámico de los sitios Guatacondo, Ramaditas, Pircas y Caserones, con el fin de reflexionar sobre el origen y desarrollo de la alfarería para fortalecer 

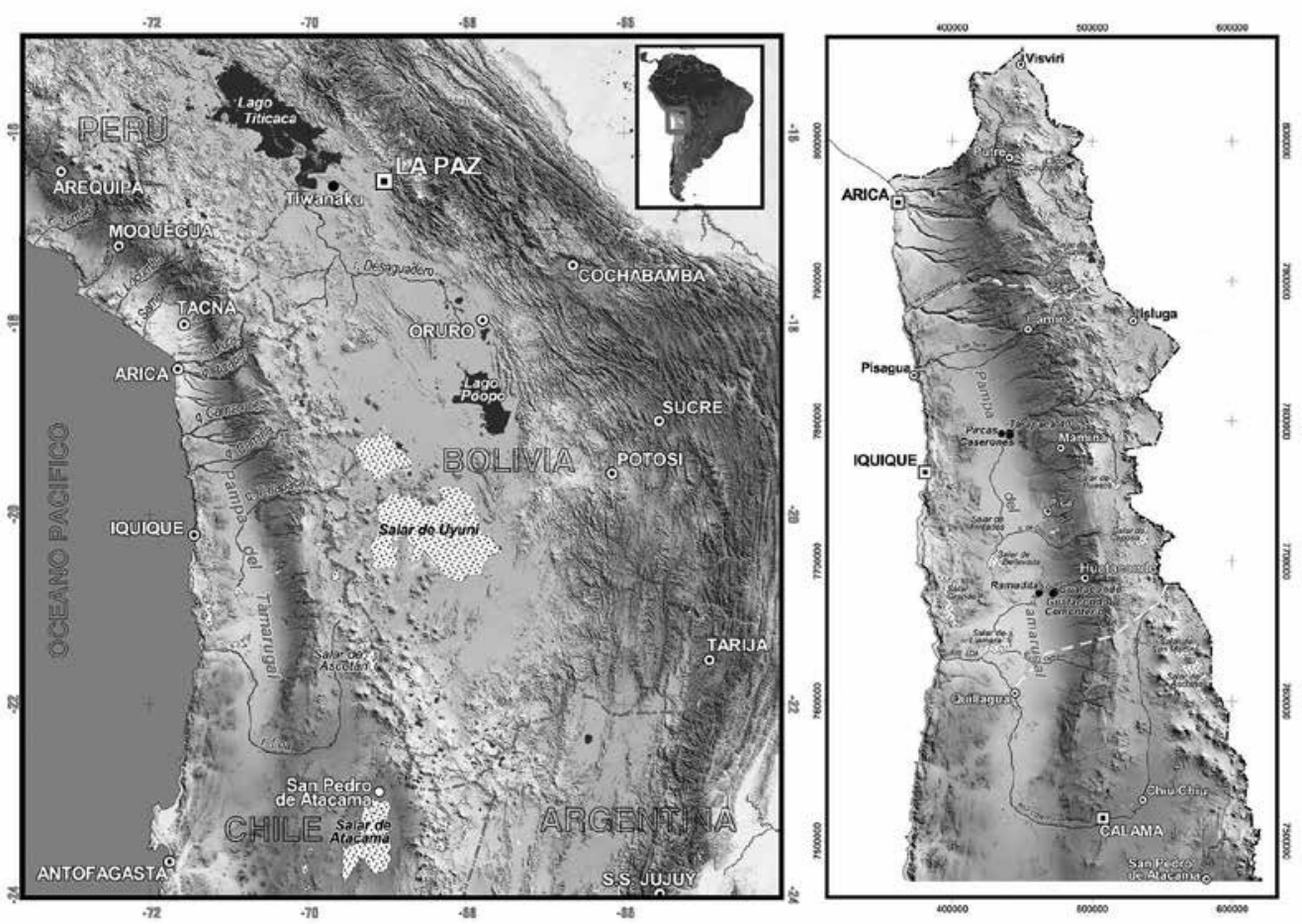

Figura 1. Mapa con ubicación de la localidad de Guatacondo, Pampa del Tamarugal, sector sur de la Región de Tarapacá, norte de Chile (propiedad de Mauricio Uribe).

la comprensión de los procesos de complejidad social que experimentaron los grupos humanos que existieron y circularon por la Pampa del Tamarugal y su costa entre los ańos 900 a.C. y 900 d.C. Para esos efectos se compararon fragmentos, se reconstruyeron formas, los tratamientos de superficie y sus pastas, junto con un primer acercamiento petrográfico, composicional y dataciones radiométricas de las muestras.

Según estas y otras evidencias, como los patrones de asentamiento, los autores propusieron que la tecnología cerámica inicial representada por el tipo Loa Café Alisado, parece desarrollarse a partir de un conocimiento profundo, aunque perteneciente a un segmento restringido de la población. Lo anterior, por ejemplo, manifiesto en la cantidad acotada de cerámica temprana, amplia distribución y la homogeneidad — tanto petrográfica como química— de las materias primas con que se elaboró una variedad limitada de vasijas con funcionalidad precisa, sugiriendo un origen y producción local de la alfarería tarapaqueña.

\section{Cerámicas del Período Formativo Temprano en el norte de Chile}

Retomando estos trabajos previos y el origen de la alfarería local, para este estudio son de interés los tipos Loa Café Alisado (LCA) y Los Morros (LMS), los que aparecen constantemente mencionados en las tipologías representativas del Período Formativo Temprano en el norte de Chile (Uribe y Ayala 2004; Uribe 2006).

El tipo Loa Café Alisado (LCA) corresponde a una tradición cerámica característica de Tarapacá, pero que también se extiende por toda la cuenca del río Loa, e incluso hacia la costa desértica (Correa et al. 2019). Las vasijas de este tipo son principalmente restringidas y no restringidas, fueron hechas con técnica de enrollamiento anular y cocidas en ambiente oxidante bien controlado, 


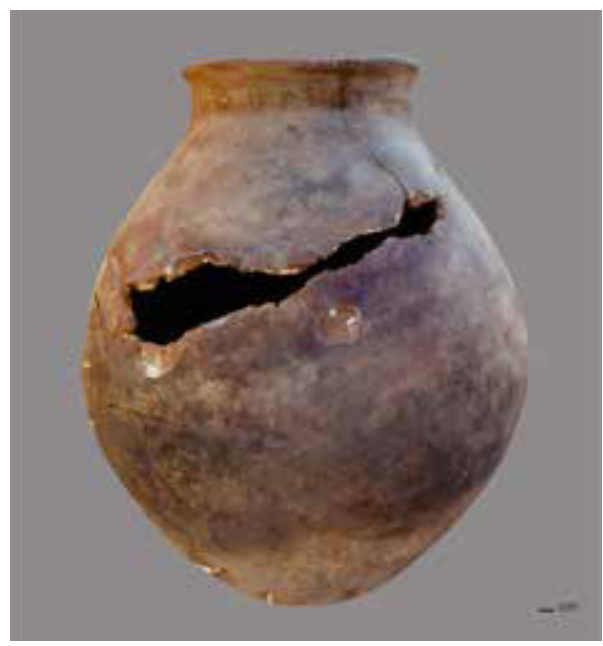

Figura 2. Vasija representativa del tipo cerámico Loa Café Alisado (fotografía de Mauricio Uribe).

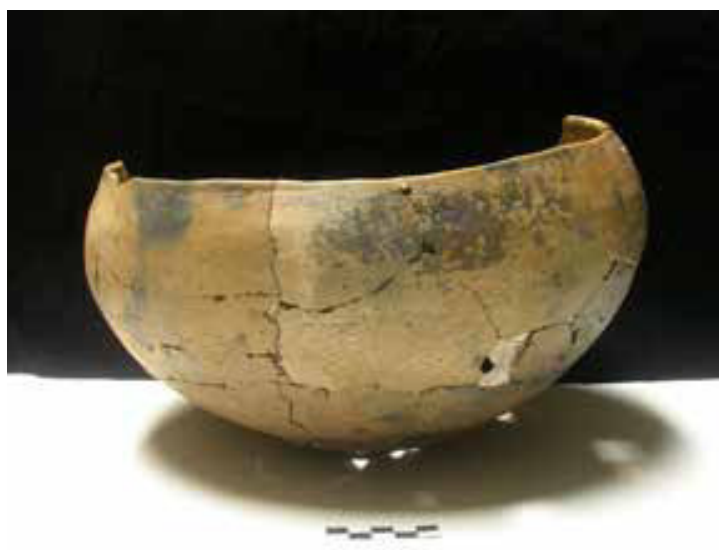

Figura 3. Vasija representativa del tipo cerámico Los Morros (fotografía de Mauricio Uribe).

con un característico «borde en coma» (evertido y grueso), (Fig. 2). Sus superficies se distinguen por un rasmillo fino y la probable aplicación de un revestimiento rojo delgado y disparejo. Su composición fue analizada mediante microscopio óptico de luz reflejada, dando como resultado una petrografía típica de pastas graníticas de aspecto arenoso, con inclusiones minerales densas en clastos blancos (Uribe y Vidal 2015). En momentos del Formativo Tardío, en Tarapacá, este tipo aparece recurrentemente asociado a los tipos Quillagua-Tarapacá; mientras que hacia el Loa y San Pedro de Atacama, es frecuente encontrarlo asociado a cerámica pulida típica de San Pedro de Atacama. Lo anterior, da cuenta de una amplia circulación e indiscutible interacción cultural entre ambas regiones durante distintos momentos del Formativo (Uribe y Ayala 2004; Uribe 2006; Uribe y Vidal 2012).

Inicialmente, el tipo Loa Café Alisado fue fechado por termoluminiscencia en el río Loa (superior) hacia 555 ańos d.C. (Sinclaire et al. 1998); sin embargo, evidencias nuevas confirman que se desarrolló antes. Dataciones del mismo tipo cerámico en la localidad de Quillagua (Loa inferior) lo sitúan entre los 730 y 530 ańos a.C. (Agüero et al. 2001, 2006). Esto posiciona al tipo Loa Café Alisado como una de las cerámicas más tempranas del desierto de Atacama, con un origen probable en Tarapacá, entre la Pampa del Tamarugal y el curso inferior del río Loa, alcanzando lugares tan alejados como los oasis del salar de Atacama (Uribe 2006; Uribe y Vidal 2012).

Por otro lado, el tipo Los Morros y sus variantes (A, B1 y B2) han sido atribuidos a manifestaciones foráneas (trasandinas), a pesar de ser muy recurrentes en el desierto de Atacama (Benavente 1981; Thomas et al. 1988-1989). Se caracterizan por vasijas restringidas simples y pulidas, con pastas de aspecto tosco y grueso (Fig. 3), distinguiéndose tres variedades de apariencia volcánica, distintas de los patrones locales posteriores donde predominan las pastas graníticas. La variante Los Morros A (LMS-A) consiste en pastas volcánicas con inclusiones no plásticas grises, tabulares y muy gruesas correspondientes a basaltos, de 3 hasta 10 milímetros de espesor. Los Morros B1 (LMS-B1), a diferencia de la variante A, presenta pastas con clastos blancos como cuarzo y sílices igualmente gruesos, aunque en general no superiores a 4 y 5 milímetros, además de micas o biotitas. Los Morros B2 (LMS-B2) es similar a LMS-B1, pero los ejemplares son más finos, delgados y a veces ricos en micas (Uribe 2006).

En superficie, su color es parejo a disparejo de tonalidades café, gris rojiza o roja grisácea, destacando aquellas cafés anaranjadas en Los Morros B1. Según estas tonalidades se infiere una cocción 


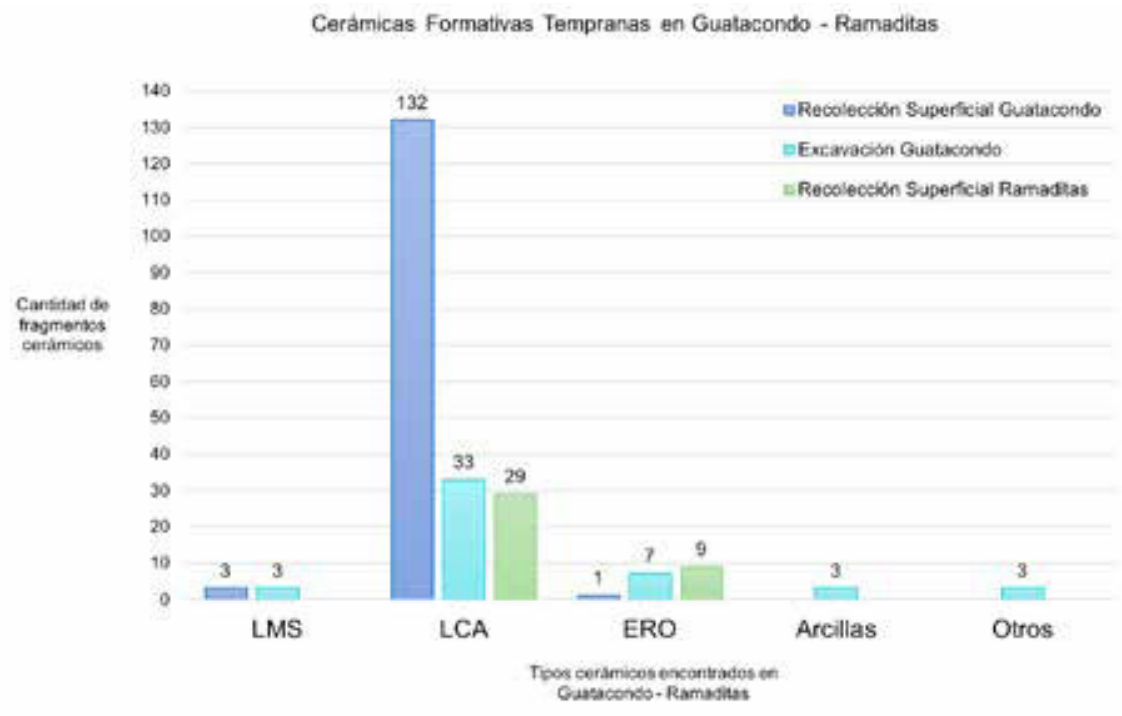

Figura 4. Gráfico con tipos cerámicos y frecuencias absolutas de las muestras recolectadas en superficie y en excavaciones de la localidad de Ramaditas (Uribe y Vidal 2012).

oxidante irregular, alcanzando temperaturas altas, pero cuya irregularidad incide en una compactación y dureza variable de las distintas pastas identificadas (Uribe 2006). Estas pastas presentan gran cantidad de cavidades por la pérdida de los clastos más gruesos debido a la fragmentación por uso doméstico y alta movilidad, dejando una fractura irregular e incluso de apariencia deleznable.

Los Morros A ha sido fecha.do por termoluminiscencia entre 960 a.C. hasta 341 d.C., aunque su mayor incidencia se ubicaría entre 550 y 165 años a.C. (Benavente 1981; Sinclaire et al. 1998; Thomas et al. 2002). Por lo tanto, se trataría de otra alfarería temprana del desierto de Atacama, análoga a las cerámicas de la fase Vega Alta del Loa Medio y Tilocalar de San Pedro de Atacama (Pollard 1982; Núñez 1994), con fechados radiocarbónicos entre el 1190 y 470 a.C. (Núñez 1995). Nuestras dataciones de termoluminiscencia ubican al tipo Los Morros entre los ańos 870 y 360 a.C. (Uribe 2006), a partir de muestras de Poconche y Yaye-Corral de Toros en los oasis de San Pedro de Atacama. A diferencia del tipo Loa Café Alisado, esta alfarería se puede definir como propia de las tierras altas circumpuneñas, de amplia circulación y nexos de origen en la vertiente oriental andina.

\section{Cerámicas tempranas de la localidad de Guatacondo}

En Ramaditas y Guatacondo, ubicados en la quebrada de Guatacondo, Uribe y Vidal (2012) analizaron 223 fragmentos en total; 136 y 38 de recolección superficial respectivamente, y 49 de diferentes unidades de excavación en Guatacondo (Tabla 1).

Dentro de la recolección superficial, en Guatacondo el tipo Loa Café Alisado aparece como el de mayor frecuencia (Fig. 4), con 132 fragmentos recolectados, acompañado por solo tres fragmentos del tipo Los Morros y uno de cerámica erosionada. Por otro lado, la recolección superficial en Ramaditas comprende 29 fragmentos Loa Café Alisado y nueve fragmentos erosionados. Prácticamente, el total de la muestra representa ocupaciones del Formativo Temprano, tratándose de un sistema de asentamiento monocomponente, bajo predominio tarapaqueño, con algunas expresiones puneñas y nulos vínculos con períodos más tardíos de la región. 
Tabla 1. Proveniencia de las muestras cerámicas del sitio Guatacondo 1, según estructura y origen estratigráfico (superficie y/o excavación) (Tomado de Uribe y Vidal 2012).

\begin{tabular}{|c|c|c|c|c|}
\hline \multicolumn{3}{|c|}{ Guatacondo } & \multicolumn{2}{|c|}{ Ramaditas } \\
\hline Estructura & Rec. superficial & Excavación & Estructura & Rec. superficial \\
\hline 3 & 3 & 31 & 9 & 5 \\
\hline 27 & 3 & & 10 & 15 \\
\hline 35 & 2 & & 41 & 1 \\
\hline 51 & 3 & & 73 & 4 \\
\hline 64 & 1 & 1 & Campo 1 & 13 \\
\hline 69 & 2 & & Subtotal & 38 \\
\hline 85 & 3 & & & \\
\hline 93 & 5 & & & \\
\hline 94 & 1 & & & \\
\hline 99 & 7 & 33 & & \\
\hline 106 & 1 & 1 & & \\
\hline 132 & 2 & & & \\
\hline 153 & 1 & 4 & & \\
\hline 164 & 1 & 9 & & \\
\hline 172 & 2 & & & \\
\hline 174 & 99 & & & \\
\hline Subtotal & 136 & 49 & & \\
\hline
\end{tabular}

En el caso de las muestras de las excavaciones en Guatacondo, estas se obtuvieron de siete recintos, donde se distinguieron hasta siete estratos y algunos rasgos específicos. El sitio presenta una pequeña cantidad de material, donde el $12.24 \%$ proviene de los niveles superficiales, $81.63 \%$ en niveles intermedios y solo un $6.12 \%$ en niveles inferiores (Tabla 2). Estadísticamente, se repite el mismo patrón de la recolección superficial; donde el tipo Loa Café Alisado es el más abundante con 33 fragmentos, aparece en todos los recintos y en seis de siete estratos excavados. Por debajo, el tipo LMS aparece con tres fragmentos, mientras que el resto del material corresponde a otros tipos indeterminados, siete fragmentos erosionados y tres restos de arcillas (Fig. 4).

Para fines de nuestro estudio, hemos sumado a los sitios de Ramaditas y Guatacondo, aquellos de Guatacondo 2, Guatacondo 4 y Guatacondo Cementerio. Por lo tanto, contamos con una muestra cerámica mayor donde el tipo Loa Café Alisado representa el $87 \%$ del total de la muestra y Los Morros un 3\%, seguido por fragmentos erosionados con un 8\%, restos de arcillas trabajadas con el $1 \%$ y otros no identificados con el 1\% (Fig. 5). Todo lo anterior confirma el carácter temprano de los asentamientos del tramo bajo de la quebrada, cuyo material de superficie como de excavaciones representan ocupaciones del Formativo entre los años 900 a.C. y 200 d.C., sin intervenciones posteriores. Junto con ello, destacamos que en Guatacondo claramente concurren dos expresiones de alfarería temprana en el mismo espacio. Esto sugiere vínculos entre poblaciones tarapaqueńas y atacameńas, ya sea por movimiento de poblaciones, intercambio de piezas o conocimientos; o simplemente, la existencia de tradiciones independientes, pero con manifestaciones similares debido a condiciones ambientales, temporales y sociales equivalentes. 
Tabla 2. Distribución estratigráfica de los tipos cerámicos hallados en Guatacondo 1 (Modificado de Uribe y Vidal 2012).

\begin{tabular}{|c|c|c|c|c|c|c|c|}
\hline \multirow[b]{2}{*}{ Estratos } & \multicolumn{7}{|c|}{ Guatacondo 1} \\
\hline & Capa & LCA & LMS & Otros & Arcillas & Erosinado & Tota \\
\hline \multirow{2}{*}{ Superficiales } & 1 & 4 & & & & & 4 \\
\hline & 1 (RASGOS 1) & 2 & & & & & 2 \\
\hline \multirow{7}{*}{ Intermedios } & 2 & 7 & & & & & 7 \\
\hline & 2 (RASGO 1) & 1 & & & & & 1 \\
\hline & $3 \mathrm{~A}$ & 14 & 1 & 3 & & 7 & 25 \\
\hline & $3 \mathrm{~B}$ & 1 & & & & & 1 \\
\hline & $4(50-60 \mathrm{~cm})$ & 2 & 2 & & & & 4 \\
\hline & 4 & 1 & & & & & 1 \\
\hline & 5 & 1 & & & & & 1 \\
\hline \multirow[t]{2}{*}{ Inferior } & 7 & & & & 3 & & 3 \\
\hline & Total general & 33 & 3 & 3 & 3 & 7 & 49 \\
\hline
\end{tabular}

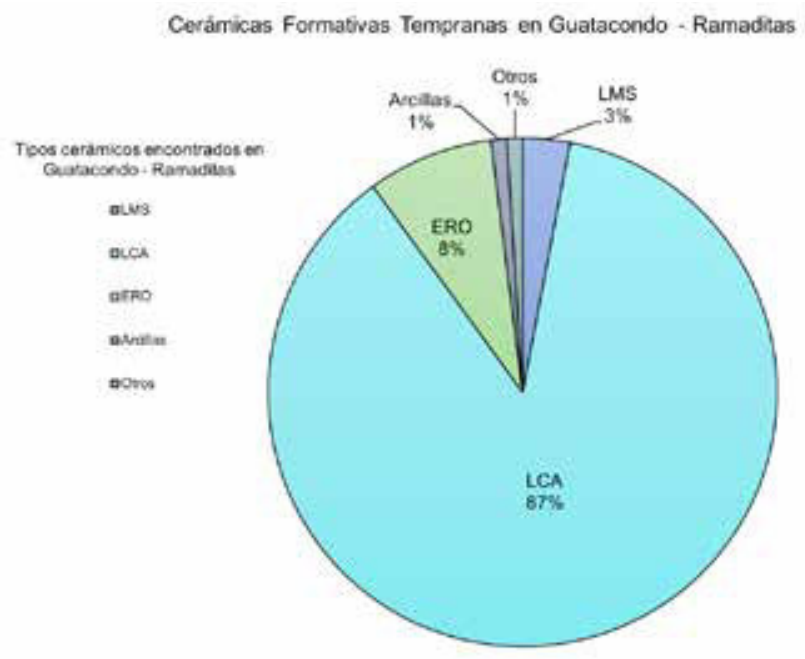

Figura 5. Gráfico con tipos cerámicos y frecuencias absolutas de las muestras recolectadas en superficie y en excavaciones de la localidad de Guatacondo (Uribe y Vidal 2012).

Nuestra intención, por lo tanto, es evaluar y profundizar nuestras hipótesis sobre la cerámica arqueológica de Tarapacá combinando la petrografía tradicional con la aplicación de nuevas técnicas como el análisis petrográfico automatizado.

\section{Metodología: análisis mineralógico automatizado QEMSCAN}

La técnica utilizada en esta investigación es el análisis mineralógico automatizado mediante QEMSCAN, acrónimo inglés de Quantitative Evaluation of Minerals by Scaning Electron Microscopy. 
Concretamente, es un microscopio electrónico de barrido modificado, tanto en sus elementos físicos y mecánicos como en el software o programa computacional de lectura de datos. QEMSCAN es un sistema configurado para medir la variabilidad mineralógica a base de la información química en escala micrométrica, originalmente desarrollado para proporcionar datos mineralógicos rápidos, automatizados y totalmente cuantitativos para la industria minera (Pirrie et al. 2004; Menzies et al. 2015; González-Maurel et al. 2019; Uribe et al. 2019).

En este caso no se usan láminas delgadas ${ }^{1}$ como en los estudios petrográficos tradicionales. Los fragmentos fueron montados en briquetas pulidas. Un grupo de seis briquetas necesita un tiempo de seis a ocho horas para su preparación; el cual se divide en etapa de montaje, luego corte-pulido y finalmente cobertura de carbón. Sin embargo, este tiempo variará según la fragilidad de las muestras montadas, ya que la etapa de corte-pulido toma más tiempo en fragmentos más deleznables. Para mayor información sobre la preparación de muestras en cerámicas, revisar Riera-Soto 2019.

Para la identificación se realiza un mapeo de distribución bidimensional (2D) y cuantificación de fases inorgánicas a partir de la combinación de emisiones de electrones retrodispersados (BSE) y rayos $\mathrm{X}$, que caracterizan la composición elemental de las fases mineralógicas reconocidas en el punto a analizar. Las lecturas de QEMSCAN están basadas en la obtención automatizada de EDS o espectros de la energía dispersada de rayos X característicos, sobre cientos de miles de puntos de análisis recolectados en un orden de milisegundos. La clasificación de fases mineralógicas se realiza mediante la clasificación de cada espectro EDS en una lista composicional jerárquica y descendente conocida como lista SIP. Para la obtención de datos de alta calidad tanto en cerámicas arqueológicas como en roca, la base de datos SIP del QEMSCAN debe ser modificada. Esto es lo que permite realizar la identificación y cuantificación automatizada de rangos y definiciones elementales que pueden ser asociadas a fases sólidas inorgánicas, ya sean minerales, aleaciones, escorias, etc. (Pirrie et al. 2004). Por su parte, la imagen BSE que lleva la información composicional es utilizada para discriminar entre la resina Epofix y el grafito que cubre la muestra, con el fin de especificar entradas en la lista SIP y establecer umbrales de aceptación o rechazo de las partículas identificadas (Pirrie et al. 2004).

Los resultados del análisis son imágenes pixeladas en 2D y color falso, donde cada píxel conserva su información elemental y brillo BSE, lo que facilita el posterior procesamiento de datos por medio del programa iDiscover v5.3.2. El uso del software permite la construcción de filtros personalizados para cuantificar abundancias minerales que se necesitan para cada estudio. Hasta este momento se pueden identificar fases minerales, tanto la composición general de la matriz arcillosa como la composición mineralógica de las inclusiones no plásticas, además de incluir la cuantificación de la porosidad. Todo esto se presenta en los mapas mineralógicos, donde se clasifican todas las partículas de acuerdo con criterios de forma, tamaño y textura, entre otros. Mientras tanto, la identificación de líticos y vidrio volcánico se hace visualmente a partir de los mapas mineralógicos y las texturas de cada uno de ellos. En cuanto a la identificación de líticos o compuestos amorfos (v.g., vidrio volcánico), nuestro equipo está avanzando en la individualización automatizada de estos, pero para eso es necesario la calibración de algunos filtros, lo que requiere de tiempo.

Una de las ventajas de esta técnica es la precisión en el reconocimiento de las fases minerales y porosidad, así como la cuantificación automática de los datos. La calidad de la lectura dependerá de la concentración de puntos analizados, es decir, mientras existan más puntos irradiados de un área, mayor será la calidad y definición de los mapas mineralógicos generados. No obstante, a mayor calidad, también mayor tiempo de análisis; por lo cual, para obtener un mapa mineralógico bien definido el análisis puede tomar entre una a dos horas y media por fragmento. Por otro lado, la mayor desventaja de la técnica es que para el reconocimiento de todas las fases mineralógicas se debe contar con una SIP modificada, ya que originalmente QEMSCAN tiene una SIP que reconoce minerales de mena y no minerales de rocas, los que son de interés específico para la petrografía cerámica (rocas sin mineralización). 


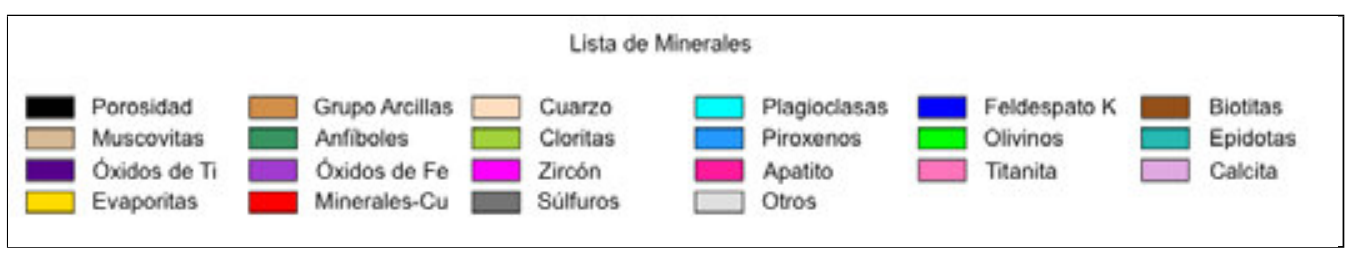

Figura 6. Listado guí de minerales o grupos minerales, seleccionados manualmente en el programa iDiscover para entender los mapas mineralógicos.

En esta oportunidad se tomaron en cuenta 20 minerales o grupos minerales, además de la porosidad y una categoría «otros», la cual agrupa todas las demás fases minerales que son identificadas por QEMSCAN, pero que no son consideradas en la lista mineralógica, o aquellas que están bajo del umbral de detección debido a irregularidades en la superficie cerámica. Los minerales o grupos minerales considerados corresponden a grupo arcillas, cuarzos, plagioclasas, feldespatos potásicos, biotitas, muscovitas, anfíboles, cloritas, piroxenos, olivinos, epidotas, óxidos de titanio (Ti) y hierro $(\mathrm{Fe})$, zircón, titanita, calcita, evaporitas, minerales de cobre $(\mathrm{Cu})$ y sulfuros (Fig. 6). La descripción e importancia de estos minerales específicos para el análisis petrográfico de cerámica arqueológica ha sido descrito por Riera-Soto (2019), destacando que la descripción individual de cada fase mineral es primordial para la interpretación de las fuentes de las materias primas, apoyada en la lectura de los mapas geológicos y/o por la recolección de muestras geológicas en terreno.

\section{Resultados}

A continuación, presentamos los resultados del análisis QEMSCAN aplicado a la cerámica temprana de Guatacondo, los cuales son interpretados a partir de una lectura directa de los datos proporcionados por la técnica en combinación con la observación y criterios de los expertos, tanto geólogos como arqueólogos.

Con el análisis mineralógico proporcionado por QEMSCAN es posible realizar una primera caracterización mineralógica automatizada para la cerámica de los valles occidentales de los Andes centro sur, específicamente de los tipos formativos tempranos Loa Café Alisado y Los Morros de Guatacondo, presentes en la región de Tarapacá. Por lo tanto, los resultados que se entregan a continuación son el comienzo para una base de datos comparativa de las diferentes petrografías de los tipos cerámicos definidos a nivel regional y para esta parte del área andina.

En el caso del tipo Loa Café Alisado (LCA) se analizaron cinco fragmentos (G-31LCA, G-36LCA, G-41LCA, G-42LCA y G-49LCA), obtenidos en los sitios Guatacondo 2, Guatacondo 4 y Guatacondo Cementerio, de distintos niveles superficiales y estratigráficos (Tabla 3). Estos fragmentos presentan una pasta media, con una matriz arcillosa ${ }^{2}$ con abundancia cercana al $45 \%$, especialmente rica en micas (alrededor del 15\%), donde la clorita predomina sobre la biotita y muscovita. Las inclusiones no plásticas están en una abundancia del 45\% (Tabla 4) y superan excepcionalmente el tamaño de la arena media, siendo en su mayoría inferiores a $500 \mu \mathrm{m}$; principalmente son monominerales y litoclastos compuestos por cuarzos, feldespatos y en menor cantidad por anfíboles (Fig. 7). Como inclusiones accesorias se observan biotitas, óxidos de hierro y titanio. Morfológicamente, las inclusiones no plásticas varían de angulosas a subredondeadas, con una buena selección y distribución. La porosidad de los fragmentos bordea el 10\% (Tabla 4), con formas primordialmente axiales (Fig. 7). 
Tabla 3. Muestras analizadas de Ramaditas y Guatacondo, correspondientes a los tipos Loa Café Alisado y Los Morros del Periodo Formativo Temprano.

Guatacondo 1, Formativo Temprano

\begin{tabular}{|c|c|c|c|c|c|}
\hline Código & Tipo & Sitio & Estructura & Unidad & Nivel \\
\hline G-31LCA & \multirow{5}{*}{$\begin{array}{l}\text { Loa Café Alisado } \\
\text { (LCA) }\end{array}$} & Cementerio & & & Superficial \\
\hline G-36LCA & & Guatacondo 2 & 2 & & Superficial \\
\hline G-41LCA & & Guatacondo 4 & 2 & & Superficial \\
\hline G-42LCA & & Guatacondo 4 & 2 & & Superficial \\
\hline G-49LCA & & Guatacondo 4 & 3 & 7 & Superficial \\
\hline G-33LMS & \multirow{6}{*}{ Los Morros (LMS) } & Guatacondo 2 & 1 & & Superficial \\
\hline G-34LMS & & Guatacondo 2 & 1 & & Superficial \\
\hline G-38LMS & & Guatacondo 2 & 1 & 5 & $2 \mathrm{~A}$ \\
\hline G-39LMS & & Guatacondo 2 & 1 & 5 & $2 \mathrm{~B}$ \\
\hline G-45LMS & & Guatacondo 4 & 2 & & Superficial \\
\hline G-53LMS & & Guatacondo 4 & 2 & 6 & Superficial \\
\hline
\end{tabular}

Por otra parte, debido a la muestra acotada, el tipo Los Morros se consideró como un conjunto completo, sin las variantes descritas para el río Loa y San Pedro de Atacama. De este modo, se analizaron seis fragmentos (G-33LMS, G-34LMS, G-38LMS, G-39LMS, G-45LMS, G-53LMS), obtenidos de los sitios Guatacondo 2 y Guatacondo 4, en distintos niveles superficiales y estratigráficos (Tabla 3). Los fragmentos comparten una pasta fina-media, con una matriz arcillosa granulosa con abundancia entre 40-50\%. Las inclusiones no plásticas se encuentran en una abundancia entre $60-50 \%$ y presentan un tamańo no superior a la arena media $(500 \mu \mathrm{m})$, (Tabla 4$)$; las que corresponden principalmente a monominerales compuestos por feldespatos, cuarzos, anfíboles, piroxenos y en menor cantidad inclusiones líticas de origen volcánico y otras de origen intrusivo. En aquellas de composición volcánica se reconoce una masa fundamental, posiblemente compuesta por vidrio volcánico desvitrificado ${ }^{3}$, la que contiene fenocristales de plagioclasa y minerales máficos (Fig. 8); las de origen intrusivo, en tanto, se reconocen por el contacto de plagioclasas con minerales máficos. En el caso de las muestras G-33LMS, G-39LMS y G-45LMS se pueden identificar olivino como inclusiones trazas, además de óxidos de hierro y titanio en todos los ejemplares. Morfológicamente, las inclusiones no plásticas varían de angulosas a subredondeadas, con una selección y distribución moderada. La porosidad de estos fragmentos es variable (Tabla 4) y se presenta de manera axial (Fig. 8).

\section{Discusión}

\subsection{Loa Café Alisado}

A partir de los atributos mineralógicos observados en este conjunto cerámico, una de las características más relevantes en la matriz arcillosa es la abundancia de cristales de clorita $(<15 \mu \mathrm{m})$ y la homogeneidad de las inclusiones no plásticas. La clorita presenta su mayor abundancia como parte de la matriz arcillosa, por lo mismo, le otorga un color verde característico en los mapas mineralógicos de estas pastas (Fig. 6). Una matriz arcillosa rica en clorita y biotita podría indicar que 


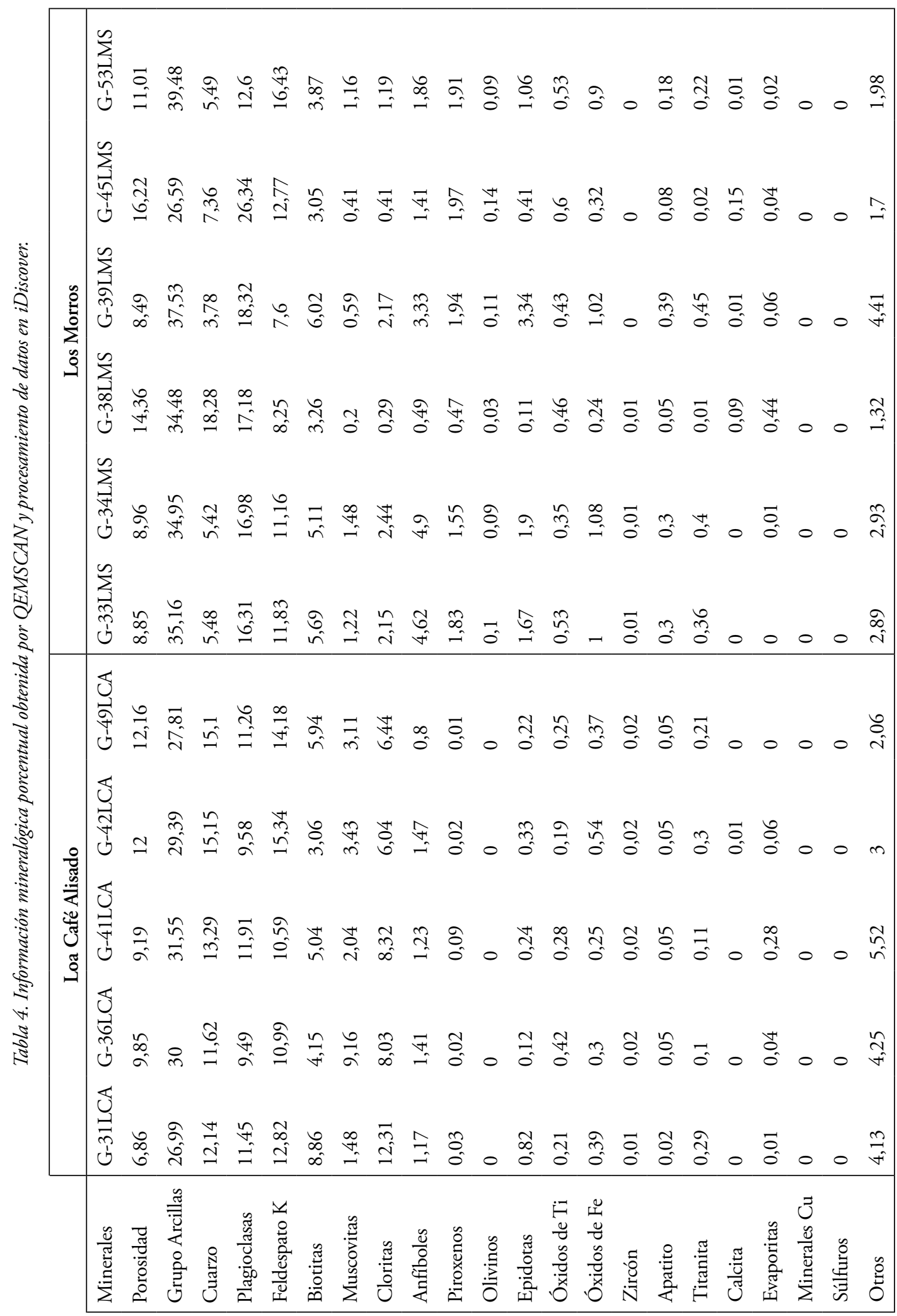




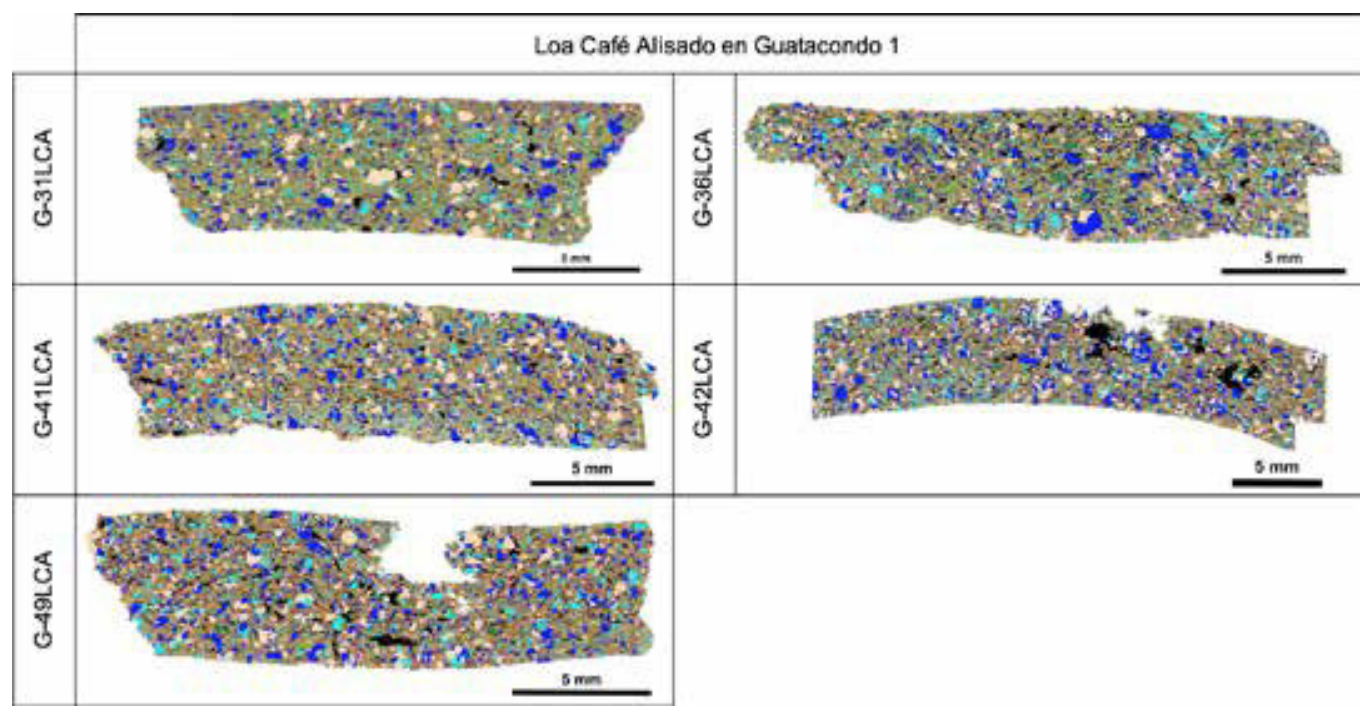

Figura 7. Mapas mineralógicos de muestras del tipo Loa Café Alisado (LCA), recuperadas en Guatacondo (ver simbología en Figura 6).

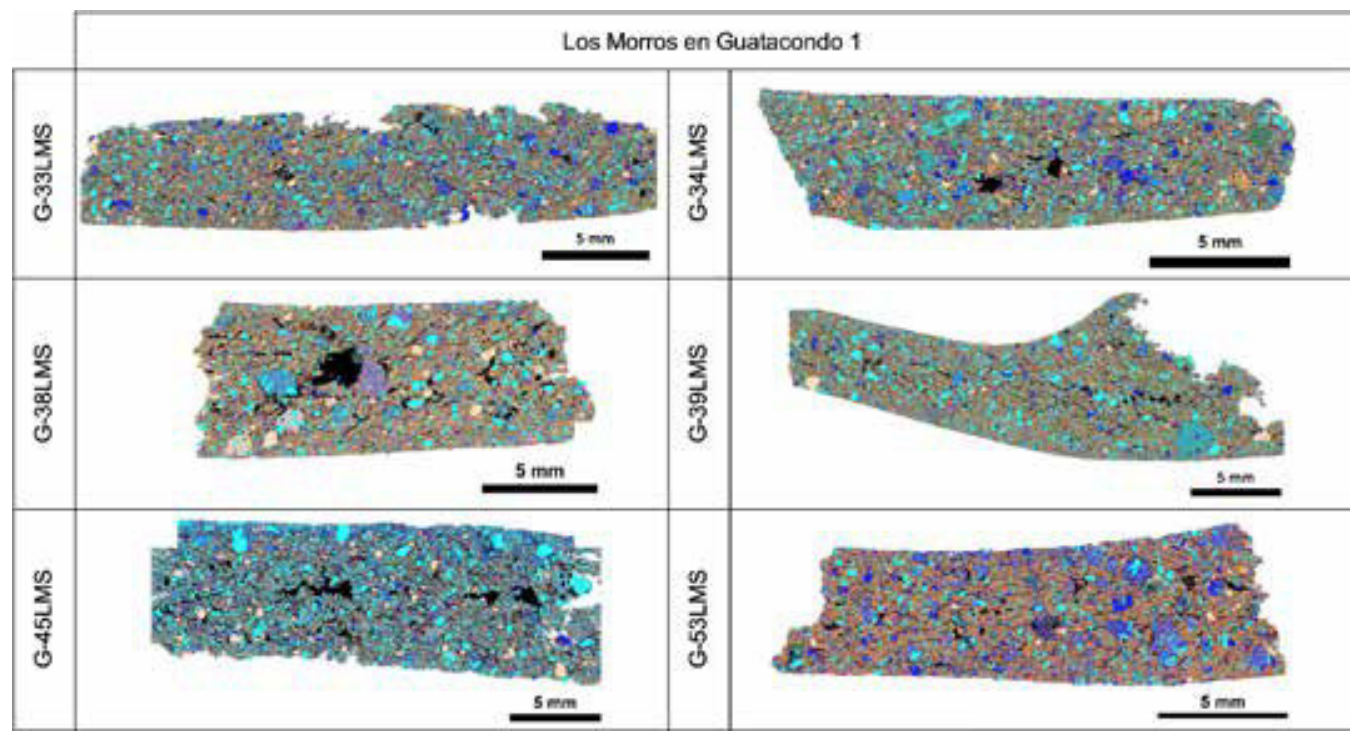

Figura 8. Mapas mineralógicos de muestras del tipo Los Morros (LMS), recuperadas en Guatacondo (ver simbología en Figura 6).

esta fracción proviene de la erosión de rocas ricas en minerales máficos, de los cuales pudo derivar la clorita como alteración de tales minerales o de la misma biotita. Nuestra observación queda respaldada por la presencia de inclusiones de anfíboles uralitizados ${ }^{4}$ a clorita, los que en algunos casos comparten un intercrecimiento con las inclusiones mayores de cuarzo y feldespatos. Por otro lado, la composición y textura de estos litoclastos indican que su origen es ígneo intrusivo, es decir, provienen de una formación geológica del tipo granítica. La gran similitud en la composición de 
los litoclastos entre los fragmentos indica que se trata de una misma fuente geológica de aprovisionamiento. Con esto no pretendemos decir que se trata exactamente de la misma fuente, sino de una unidad geológica, probablemente aluvial o fluvial que es reconocida por proveer una calidad de materias primas en particular, donde se conserva un tipo de líticos específicos, pero varía la porción de partículas tamaño arcilla.

Además, las características morfológicas de las inclusiones no plásticas, angulosas a subredondeadas, con buena selección y distribución, junto con una porosidad bastante regular en abundancia y forma, indican que se trata de una materia prima bien seleccionada. Es decir, nos parece que se buscaba una granulometría en particular, asociada a un buen trabajo de amasado y cierto nivel de práctica del artesano (Druc y Chávez 2014), lo que permite obtener una pasta homogénea en distribución de clastos y entre los fragmentos (Fig. 7). En suma, sugiere una misma forma de hacer vasijas.

\subsection{Los Morros}

En comparación con los ejemplares Loa Café Alisado, los fragmentos Los Morros de Guatacondo poseen una mayor variabilidad entre ellos.

Los atributos mineralógicos más representativos de los seis fragmentos analizados son las inclusiones no plásticas de origen volcánico y una matriz arcillosa granulosa. Esta última característica está dada por partículas de similar apariencia a la masa fundamental de los clastos de origen volcánico, por lo cual se puede suponer que corresponde a vidrio volcánico desvitrificado. Ahora bien, la variación composicional particular notada en las matrices arcillosas da a entender que se trata de fuentes de aprovisionamiento que pueden ser distintas espacial, temporal y/o composicionalmente, aunque todas con un componente volcánico claro determinado por las inclusiones no plásticas. La escasa presencia de cuarzo y notoria existencia de minerales máficos con plagioclasas, permite confirmar que las fuentes de aprovisionamiento de materias primas en estos casos son de carácter volcánico; probablemente andesitas, por el modo de ocurrencia de algunas inclusiones no plásticas dentro de la masa fundamental, y reafirmado por la existencia de olivino como mineral traza. Estas fuentes de aprovisionamiento, destacamos, varían espacial y/o temporalmente dentro de un mismo ambiente geológico volcánico.

En definitiva, la granulometría del tipo Los Morros aparece como una característica primordial. Uribe (2006) ha descrito esta cerámica en el salar de Atacama como una alfarería variada y rica en inclusiones gruesas con respecto a la matriz arcillosa, lo que se comprueba con las muestras analizadas en este estudio. Además, las inclusiones no plásticas con sus formas angulosas a subredondeadas, junto a una selección y distribución moderada, más la porosidad variable en forma y abundancia, podrían indicar que se trata de modos diferentes de hacer vasijas.

\subsection{Otras observaciones}

En particular, para las muestras G-38LMS y G-41LCA se pudieron identificar poros rellenos con minerales evaporíticos, correspondiente a un relleno sutil, aunque detectable. Este grupo de minerales corresponde a las sales solubles que son muy comunes, tanto en el suelo, como el agua del Norte árido de Chile. El relleno de estos poros podría deberse al almacenamiento de líquidos. Estos minerales pudieron precipitar de dos maneras, ya sea por la cocción de líquidos o por el almacenamiento prolongado de los mismos con su posterior evaporación. Todo esto evidencia que la principal funcionalidad de estas vasijas, pudo estar asociada a la contención de líquidos. 


\section{Conclusiones}

En primer lugar, podemos concluir que existe equivalencia y complementariedad entre lo observado de manera macroscópica y cualitativa por parte de los arqueólogo/as (Uribe y Vidal 2015; Uribe et al. 2019), frente a la apreciación microscópica, automatizada y cuantitativa obtenida por los geólogos a través de la aplicación de QEMSCAN. Se comprueba que, petrográficamente, los tipos cerámicos Loa Café Alisado y Los Morros corresponden a tradiciones alfareras y estilos tecnológicos distintos.

Para el tipo Loa Café Alisado, según la mineralogía y las texturas de intercrecimiento de las inclusiones mayores y la clorita presentes en su matriz arcillosa, se puede catalogar como un grupo petrográfico bien definido en Guatacondo. La materia prima de esta cerámica pudo ser obtenida de una misma fuente espacial y/o temporal, lo cual genera una composición de pasta muy similar. Esta fuente, a su vez, podría ser el producto de la erosión mecánica de una formación ígnea intrusiva granítica transportada por flujos ricos en arcillas de composición variada. Esto es posible por la naturaleza aluvional de los causes fluviales de la Pampa del Tamarugal que son muy ricos en arcillas (Blanco y Tomlinson 2013). Las materias primas análogas y el trabajo de amasado de la pasta son muy similares en todos los fragmentos analizados, por lo que se trataría de un mismo modo de hacer cerámicas, desde la búsqueda de la materia prima hasta la manera de construir vasijas. Por lo anterior, podemos suponer y proponer que se trató de una producción local desarrollada por las poblaciones de la Pampa del Tamarugal.

En tanto, los fragmentos del tipo Los Morros presentan una mayor variabilidad en su composición, por lo que, al contrario del Loa Café Alisado, no se puede considerar como un grupo petrográfico en Guatacondo. En efecto, todos los fragmentos analizados comparten un componente volcánico en sus materias primas, pero además la disparidad entre sus matrices arcillosas granulosas denota diferentes fuentes de aprovisionamiento espaciales y/o temporales en un mismo ambiente geológico foráneo a la Pampa del Tamarugal. Lo anterior, es elocuente en la distinción de variedades dentro de la misma clase cerámica (Uribe 2006). En consecuencia, asumimos y proponemos que se trata de una alfarería en la que se trasfiere el conocimiento sobre materias primas y construcción de vasijas, pero que va interactuando con diferentes lugares de aprovisionamiento, de acuerdo con una amplia circulación y origen esencialmente puneño. Por lo mismo, es probable, pudo llegar a través de redes de intercambio a larga distancia o por la presencia eventual de poblaciones de tierras altas en Guatacondo.

En suma, la presencia de los dos tipos cerámicos Loa Café Alisado y Los Morros en Guatacondo, al sur de Tarapacá y al norte del río Loa, da cuenta de dos situaciones o sistemas de acceso y desarrollo de la alfarería durante el Formativo Temprano regional. Uno que indica la existencia de una producción local, marcada por la calidad de materia prima en particular, y otro derivado de redes de movilidad e intercambio a larga distancia, donde el conocimiento es proporcionado por la circulación; sin negar la posibilidad de la convivencia de poblaciones y producciones distintas en Guatacondo. En cualquier caso, la presencia moderada del material cerámico en los asentamientos en cuestión, sugiere que se trató de una tecnología acotada, probablemente en conocimiento y experticia de unos pocos miembros de sus comunidades. Esto, por otra parte, indica que la importancia socioeconómica de la alfarería todavía se encontraba en ciernes durante el Formativo Temprano de esta parte de los Andes centro sur.

\section{Agradecimientos}

Los autores de este artículo agradecemos a la Unidad de Equipamiento Científico MAINI de la Universidad Católica del Norte (UCN), por la colaboración en la preparación de muestras y generación de datos, en especial a las técnicas profesionales Pamela Fonseca, María Eugenia González y Carolina Ossandón. También agradecemos el apoyo del proyecto FONDECYT 1181829. 


\section{Notas}

${ }^{1}$ La técnica QEMSCAN permite la lectura tanto de briquetas pulidas como de láminas delgadas. En el caso de estas últimas, el material a analizar debe tener un espesor de al menos $70 \mu \mathrm{m}$ y no estar cubiertos por ningún tipo de resina o cubre objeto.

${ }^{2}$ Este estudio considera matriz arcillosa a todas las partículas menores a $15 \mu \mathrm{m}$ (Courtois 1976), ya que ese es el límite que se utiliza en la bibliografía (v.g. Druc y Chávez 2014).

${ }^{3}$ La composición química del vidrio volcánico es bastante similar a la del feldespato potásico debido a que tanto el feldespato potásico como el vidrio volcánico corresponden a la última etapa de la evolución magmática, con la diferencia que el primero logra su cristalización como mineral y el segundo es amorfo. Esta composición se verificó en el programa iDiscover utilizando una lista de minerales para rocas volcánicas. Además, en los mapas mineralógicos se puede diferenciar directamente el feldespato potásico del vidrio volcánico desvitrificado, porque en el vidrio la desvitrificación se presenta con la aparición de minerales de arcillas junto con fenocristales de diferentes minerales. Estos dos componentes de las inclusiones no plásticas fueron revisados por una lista mineralógica para rocas volcánicas confeccionada por el Dr. Menzies para iDiscover.

${ }^{4}$ Uralitización es la «hidratación» de los minerales máficos por medio de hidrólisis, mecanismo por el cual un piroxeno o anfíbol puede alterarse a biotita y/o clorita, dependiendo del grado de alteración.

\section{REFERENCIAS}

\section{Agüero, C., P. Ayala, M. Uribe, C. Carrasco y B. Cases}

2006 El Período Formativo desde Quillagua, Loa Inferior (norte de Chile), en: H. Lechtman (ed.), Esferas de interacción prehistóricas y fronteras nacionales modernas: los Andes Sur Centrales, 73-120, IEP-IAR, Lima.

Agüero, C., M. Uribe, P. Ayala, B. Cases y C. Carrasco

2001 Ceremonialismo del período Formativo en Quillagua, Norte Grande de Chile, Boletín de la Sociedad Chilena de Arqueología 32, 24-34.

Blanco, N. y A Tomlinson

2013 Carta Guatacondo. Región de Tarapacá, Servicio Nacional de Geología y Minería, Serie Geología Básica, mapa 1:100.000. Santiago.

Benavente, A.

1981 Chiu Chiu 200: un campamento de pastores, tesis de licenciatura, Departamento de Antropología, Universidad de Chile, Santiago.

Correa, I., F. Gallardo, M. Uribe, E. Echenique, J.F. Blanco, S. Flewett, M.T. Boulanger y M. Glascock

2019 Pottery from funerary mound along the arid Atacama Desert coast, Chile. Chemistry, circulation,and exchange between the inlands and coast during the Formative Period, en: M.D. Glascock, H. Neff y K.J. Vaughn (eds.), Ceramics of the indigenous cultures of South America. Studies of production and exchange through compositional analysis, 147-160, University of New Mexico Press, Albuquerque.

Courtois, L.

1976 Examen au microscope pétrographique des céramiques archéologiques, Notes at Monographies Techniques 8. CNRS, Paris.

Druc, I. y L. Chávez

2014 Pastas cerámicas en lupa digital: componentes, materiales y tecnología, Deep University Press, Wisconsin.

González-Maurel, O., Godoy, B., le Roux, P., Rodríguez, I., Marín, C., Menzies, A., Bertin, D., Morata, D. Y M. Vargas.

2019 Magmatic differentiation at La Poruña scoria cone, Central Andes, northern Chile: evidence for assimilation during turbulent ascent processes, and genetic links with mafic eruptions at adjacent San Pedro volcano, Lithos 338-339, 128-140. https://doi.org/10.1016/j.lithos.2019.03.033 


\section{Mavrakis, R.}

1985 Análisis Tipológico Morfológico de la Cerámica de Caserones (1a Región, Chile), tesis de licenciatura, Universidad del Norte, Antofagasta.

\section{Menzies, A., M. Uribe, F. Erazo, C. Ossandon y P. Fonseca}

2015 Automated mineralogical analysis of archaeological samples from northern Chile - Case Study II Ceramics, Ponencia presentada en XIV Congreso Geológico Chileno, La Serena.

Núñez, L.

1994 (1992) Emergencia de complejidad y arquitectura jerarquizada en la puna de Atacama: las evidencias del sitio Tulan-54, en: M. E. Albeck (ed.), Taller de Costa a Selva, 85-115, Instituto Interdisciplinario de Tilcara, Jujuy.

1995 Fase Tilocalar: nuevas evidencias formativas en la Puna de Atacama (Norte de Chile), en: P. LedergerberCrespo (ed.), Formativo sudamericano, una revaluación, 227-242, Ediciones ABYA-YALA, Quito.

Núńez, L. y C. Moragas

1983 Cerámica temprana en Cáñamo (costa desértica del norte de Chile): análisis y evaluación regional, Chungará Revista de Antropologia Chilena 11, 31-61.

Núñez, L. y C.M. Santoro

2011 El tránsito arcaico-formativo en la circumpuna y valles occidentales del centro sur andino: hacia los cambios «neolíticos», Chungará Revista de Antropología Chilena 43, 487-530. https://doi.org/10.4067/ S0717-73562011000300010

Pirrie, D., Butcher, A.R., Power, M.R., Gottlieb, P. y G. L. Miller.

2004 Rapid quantitative mineral and phase analysis using automated scanning electronmicroscopy (QemSCAN); potential applications in forensic geoscience, en: K. Pye y D. Croft (eds.), Forensic geoscience: Principles, techniques and applications, 123-136, Geological Society, Special Publication 232, London. https://doi.org/10.1144/GSL.SP.2004.232.01.12

Pollard, G.

1982 (1970) The cultural ecology of ceramic stage settlement in Atacama Desert, tesis de doctorado, Columbia University, University Microfilms Int., Ann Arbor.

Riera-Soto, C.

2019 Estudio petrográfico de las cerámicas formativas en la cuenca del río Vilama y oasis de San Pedro de Atacama (Norte de Chile): Materias primas en circulación, tesis de maestría, Instituto de Investigaciones Arqueológicas y Museo, Universidad Católica del Norte, San Pedro de Atacama.

Rivera, $M$.

1988- Cerámicas tempranas de la costa norte de Chile, Paleoetnológica 5, 165-172.

\section{Sinclaire, C., M. Uribe, P. Ayala y J. González}

1998 La alfarería del Período Formativo en la región del Loa Superior: sistematización y tipología, Actas del XIV Congreso Nacional de Arqueología Chilena, tomo II, 285-314, Contribución Arqueológica 5, Copiapó.

Thomas, C., C. Massone y A. Benavente

1988 Sistematización cerámica de seis yacimientos de la provincia El Loa (II Región). Paleoetnológica 5, 121151.

Thomas, C., A. Benavente, I. Cartajena y L. Contreras

2002 Una secuencia de fechados por termoluminiscencia para la localidad de Chiu-Chiu: sitios Chiu Chiu 273 Uribe, $M$. y 275, Boletín de la Sociedad Chilena de Arqueología 33-34, 84-89.

2004 Alfarería, arqueología y metodología. Aportes y proyecciones de los estudios cerámicos del Norte Grande de Chile, tesis de maestría, Universidad de Chile, Santiago.

2006 Sobre cerámica,su origen y complejidad social en los Andes del desierto de Atacama, en: H. Lechtman (ed.), Esferas de interacción prehistóricas y fronteras nacionales modernas: Los Andes Sur Centrales, 449-502, IEP-IAR, Lima. 
2009 El período Formativo de Tarapacá y su cerámica: Avances sobre complejidad social en la costa del Norte Grande de Chile (900 a.C.-800 d.C.), Estudios Atacameños 37, 5-27. https://doi.org/10.4067/S071810432009000100002

\section{Uribe, M. y P. Ayala}

2000 La alfarería de Quillagua en el contexto formativo del Norte Grande de Chile (1000 a.C.-500 d.C.), ponencia presentada en XV Congreso Nacional de Arqueología Chilena, Arica.

2004 La alfarería de Quillagua en el contexto Formativo del Norte Grande de Chile (1000 a.C.-500 d.C.), Chungará Revista de Antropología Chilena 36, 585-597. https://doi.org/10.4067/S071773562004000400006

Uribe, M. y E. Vidal

2012 Sobre la secuencia cerámica del Período Formativo de Tarapacá (900 a.C.-900 d.C.): estudios en Pircas, Caserones, Guatacondo y Ramaditas, Norte de Chile, Chungará Revista de Antropología Chilena 44(2), 209-245. https://doi.org/10.4067/S0717-73562012000200003

2015 Pottery and social complexity in Tarapacá: reviewing the development of ceramic technology in the Atacama Desert (Northern Chile), en: I. Druc (ed.), Ceramic analysis in the Andes, 15-35, Deep University Press, Blue Mounds, Wisconsin.

Uribe, M., E. Vidal, M. Glascock, A. Menzies, M. Muñoz y C. Roush

2019 A compositional characterization of ceramic production and circulation during the Formative Period in Tarapacá, Northern Chile, en: M.D. Glascock, H. Neff y K.J. Vaughn (eds.), Ceramics of the indigenous cultures of South America. Studies of production and exchange through compositional analysis, 147-160, University of New Mexico Press, Albuquerque.

Recibido: 31/01/2019

Aceptado: 31/07/2019 